\title{
Nitrogen metabolism-related enzymes in Mesembryanthemum crystallinum after Botrytis cinerea infection
}

\author{
E. GAJEWSKA ${ }^{1 *}$, E. SURÓWKA ${ }^{2}$, A. KORNAS ${ }^{3}$, and E. KUŹNIAK ${ }^{1}$ \\ Department of Plant Physiology and Biochemistry, Faculty of Biology and Environmental Protection, \\ University of Łódź, Banacha 12/16, PL - 90237 Łódź, Poland ${ }^{1}$ \\ Institute of Plant Physiology, Polish Academy of Sciences, Niezapominajek 21, PL - 30239 Kraków, Poland \\ Institute of Biology, Pedagogical University, Podchorażych 2, PL - 30084 Kraków, Poland ${ }^{3}$
}

\begin{abstract}
We compared $\mathrm{C}_{3}$ and CAM (crassulacean acid metabolism) states in Mesembryanthemum crystallinum, a facultative CAM species, with respect to the involvement of phosphoenolpyruvate carboxylase (PEPC) and nitrogen metabolismrelated enzymes in plant response to Botrytis cinerea infection. The enzyme activities were monitored both in pathogeninoculated $2^{\text {nd }}$ leaf pair and non-inoculated $3^{\text {rd }}$ leaf pair. The control activities of most studied enzymes were dependent on the mode of photosynthesis. Compared to $\mathrm{C}_{3}$ plants, those performing CAM exhibited higher PEPC, nitrate reductase (NR), and deaminating glutamate dehydrogenase (NAD-GDH) activities but lower glutamine synthetase (GS) and alanine aminotransferase (ALT) activities. Regardless of the mode of photosynthetic carbon assimilation, the plants responded to infection with enhancement of PEPC and inhibition of NR activities in the inoculated leaves. Whereas the activity of GS remained unaffected, those of all glutamate-yielding enzymes, namely ferredoxin-dependent glutamate synthase (Fd-GOGAT), aspartate aminotransferase (AST), ALT, and aminating glutamate dehydrogenase (NADHGDH) were altered after infection. However, the time-course and extent of the observed changes differed in $\mathrm{C}_{3}$ and CAM plants. In general, CAM plants responded to infection with an earlier increase in PEPC and Fd-GOGAT activities as well as later inhibition of NR activity. Contrary to $\mathrm{C}_{3}$ plants, in those performing CAM the activities of PEPC, Fd-GOGAT, NADH-GDH, and AST in the non-inoculated $3^{\text {rd }}$ leaf pair were similarly influenced by infection as in leaves directly inoculated with the pathogen. This implies that the local infection induced an alteration of carbon/nitrogen status in healthy upper leaves. This reprogramming resulting from changes in PEPC and nitrogen metabolism-related enzymes was $\mathrm{C}_{3}$ - and CAM-specific.
\end{abstract}

Additional key words: crassulacean acid metabolism, glutamate dehydrogenase, grey mould, ice plant, nitrate reductase, phosphoenolpyruvate carboxylase.

\section{Introduction}

Upon infection, the plant and the pathogen develop a complex relationship affecting almost all metabolic processes in the host cells, including carbon and nitrogen metabolism. Although the role of primary metabolism in plant defense against pathogens has evoked considerable interest, this problem still awaits clarification. As concern to nitrogen metabolism, two contrasting effects were observed during pathogenesis: 1) the nitrogen status of plant tissues influenced pathogen infection and colonization and 2) the plant nitrogen metabolism was

manipulated by the pathogen for its own benefit (Snoeijers et al. 2000, Bolton and Thomma 2008). Depending on the lifestyle of the invading pathogen, changes in nitrogen metabolism during pathogenesis can either promote plant defense or support pathogen growth and infection development (Liu et al. 2010, Seifi et al. 2013).

The main metabolic pathway by which inorganic $\mathrm{N}$ is converted into organic $\mathrm{N}$ in plants consists of sequential enzymatic reactions catalyzed by nitrate reductase

Submitted 7 November 2016, last revision 31 October 2017, accepted 12 January 2018.

Abbreviations: ALT - alanine aminotransferase; AST - aspartate aminotransferase; CAM - crassulacean acid metabolism; dai - day after inoculation; EDTA - ethylenediaminetetraacetic acid; Fd-GOGAT - ferredoxin-dependent glutamate synthase; GS - glutamine synthetase; NAD-GDH - NAD-dependent glutamate dehydrogenase; NADH-GDH - NADH-dependent glutamate dehydrogenase; NADH-GOGAT - NADH-dependent glutamate synthase; NiR - nitrite reductase; NR - nitrate reductase; PEPC - phosphoenolpyruvate carboxylase; TCA cycle - tricarboxylic acid cycle.

Acknowledgements: This work was supported by the Ministry of Science and Higher Education (grant 830/1/N-COST/2010/11).

*Corresponding author; fax: (+48) 426354423, e-mail: ewa.gajewska@biol.uni.lodz.pl 
(NR, EC 1.6.6.1), nitrite reductase (NiR, EC 1.7.7.1), glutamine synthetase (GS, EC 6.3.1.2), and glutamate synthase (GOGAT; EC 1.4.1.14 for NADH-GOGAT and EC 1.4.7.1 for ferredoxin-dependent glutamate synthase, Fd-GOGAT). In this process, NR-mediated nitrate reduction to nitrite is considered to be the rate limiting step (Masclaux-Daubresse et al. 2010).

Under environmental stresses, an additional pathway mediated by glutamate dehydrogenase (GDH, EC 1.4.1.2) is induced to control the flux of nitrogen (Cebeci et al. 2008, Miyashita and Good 2008). This enzyme catalyzes the reversible reductive amination of 2-oxoglutarate to yield glutamate and acts to avoid ammonia accumulation under stress (Skopelitis et al. 2006). However, in vivo GDH also deaminates glutamate and fuels the tricarboxylic acid cycle (TCA cycle) with 2-oxoglutarate. Alanine aminotransferase (ALT, EC 2.6.1.2) and aspartate aminotransferase (AST, EC 2.6.1.1) catalyzing the reversible transfer of amino group from glutamate to pyruvate/oxaloacetate to form 2-oxoglutarate and alanine/aspartate, respectively have also been shown to be involved in mechanisms that allow plants to survive under stress conditions (Gajewska and Skłodowska 2009, Gao et al. 2013). The aminotransferase-catalyzed amination of 2-oxoglutarate provides plant cells with glutamate which is of central importance for nitrogen remobilization during pathogenesis (Seifi et al. 2013).

In plants, the interdependence of primary carbon and nitrogen metabolism has been extensively studied and the pivotal role of photosynthesis in nitrogen assimilation is well known (Foyer and Noctor 2002, Nunes-Nesi et al. 2010, Gawronska and Niewiadomska 2015). The flexible coordination of nitrogen assimilation with carbon metabolism is considered important for a broad spectrum of physiological and developmental processes in plants (Sun et al. 2013) as well as for plant response to stressful environmental conditions, including biotic stress.

The metabolic rearrangements in plant cells during plant-pathogen interactions contribute to plant defense and they are usually manifested by downregulation of the

\section{Materials and methods}

Plant material and pathogen infection: The ice-plant (Mesembryanthemum crystallinum L.) was grown from seeds in a growth chamber as described earlier (Kuźniak et al. 2013). After the appearance of the $3^{\text {rd }}$ leaf pair, one set of plants was irrigated with $0.4 \mathrm{M} \mathrm{NaCl}$ to induce CAM (CAM plants) while another was further irrigated with water $\left(\mathrm{C}_{3}\right.$ plants). The induction of CAM in NaCltreated plants was detected by measuring diurnal malate fluctuations assumed as a hallmark of CAM (Kuźniak et al. 2013). A 12-d treatment with $0.4 \mathrm{M} \mathrm{NaCl}$ induced CAM with $\Delta$ malate in the range of $10-15 \mathrm{mM}$. Thereafter, leaves of the $2^{\text {nd }}$ leaf pairs of $C_{3}$ and CAM plants were inoculated by infiltration with Botrytis cinerea spore suspension $\left(1 \times 10^{6}\right.$ spores $\left.\mathrm{cm}^{-3}\right)$ supplemented with $5 \mathrm{mM}$ glucose and $2.5 \quad \mathrm{mM} \mathrm{KH}_{2} \mathrm{PO}_{4}$ primary metabolism, mainly photosynthesis and other processes supporting plant growth (Scharte et al. 2005, Bilgin et al. 2010). The energy and metabolite resources saved by downregulation of the primary metabolism can be diverted to defense processes. Our previous studies demonstrated that the infection-induced changes in photochemical activity could be also dependent on the type of photosynthetic carbon assimilation. In Mesembryanthemum crystallinum, a facultative $\mathrm{C}_{3}$-CAM (crassulacean acid metabolism) plant, we observed different patterns of Botrytis cinerea-induced changes in the photochemical activity in $\mathrm{C}_{3}$ and CAM plants. While in CAM plants, the photochemical activity visualized by chlorophyll fluorescence imaging was decreased, in $\mathrm{C}_{3}$ plants, it remained unchanged (Gabara et al. 2012). These infection-induced changes could alter the flow of carbon skeletons and reduced equivalents to interacting pathways of nitrate nitrogen assimilation.

Phosphoenolpyruvate carboxylase (PEPC, EC 4.1.1.31), indispensable for primary $\mathrm{CO}_{2}$ fixation in $\mathrm{C}_{4}$ and CAM plants, serves a variety of non-photosynthetic functions. In all photosynthetic plant types, PEPC is involved in the coordination of carbon and nitrogen assimilation by replenishing the TCA cycle with oxaloacetate which is converted into 2-oxoglutarate used in nitrogen assimilation (Doubnerová and Ryšlavá 2011).

The aim of this study was to analyze the effects of $B$. cinerea infection on nitrogen metabolism enzymes in leaves of $M$. crystallinum with respect to the type of photosynthetic carbon assimilation. The activities of nitrogen metabolism-related enzymes, namely NR, GS, Fd-GOGAT, GDH, ALT, and AST, as well as PEPC involved in coordination of carbon and nitrogen assimilation were monitored in plants operating in the $\mathrm{C}_{3}$ and CAM mode. This enzymatic response was studied both locally (in the inoculated leaves) and systemically (in the non-inoculated upper leaves). To our knowledge, this is the first report on the influence of infection on nitrogen metabolism-related enzymes in M. crystallinum, the model $\mathrm{C}_{3}$-CAM plant.

according to Kuźniak et al. (2013). Control plants were

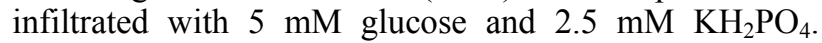
Leaves of control and infected $\mathrm{C}_{3}$ and CAM plants were taken for analyses 1, 2, and $3 \mathrm{~d}$ after inoculation (dai). We analyzed samples from the $2^{\text {nd }}$ leaf pairs (inoculated leaves) as well as from the $3^{\text {rd }}$ leaf pairs (non-inoculated upper leaves) prepared as described by Kuźniak et al. (2013). Samples were collected in the middle of the light period.

Microscopy analysis of fungal infection: Samples of infected leaf tissues $(0.5 \times 0.5 \mathrm{~cm})$ were taken from the infiltrated areas $20 \mathrm{~h}$ after inoculation, squashed in distilled water and examined under a light microscope (Leica, Wetzlar, Germany) equipped with digital camera. 
Alternatively, the samples were stained with $0.01 \%$ $(\mathrm{m} / \mathrm{v})$ trypan blue in lactophenol. The plant material was transferred into a test tube, covered with staining solution, placed in a heated water bath and boiled for one minute. The staining solution was removed by boiling in lactoglycerol (glycerol $+90 \%$ lactic acid + distilled water in volume proportions 1:2:5). The samples were mounted in glycerol, observed under the light microscope, and photographed.

Preparation of leaf extracts: The major leaf veins were removed from the leaves and the samples for biochemical analyses $(0.5 \mathrm{~g})$ were prepared from the remaining leaf lamina (Kuźniak et al. 2016). For estimation of NR and Fd-GOGAT activities, fresh tissue was homogenized $(1: 5, \mathrm{~m} / \mathrm{v})$ in the ice cold mortar using $50 \mathrm{mM}$ potassium phosphate buffer, $\mathrm{pH} 7.5$, containing $2 \mathrm{mM}$ EDTA, $10 \mathrm{mM} \mathrm{KCl}, 14 \mathrm{mM} \quad \beta$-mercaptoethanol, $1 \mathrm{mM}$ phenylmethylsulfonyl fluoride, and $3.58 \mathrm{M}$ ethylene glycol. For estimation of PEPC, GS, GDH, ALT, and AST activities, fresh tissue was homogenized $(1: 5, \mathrm{~m} / \mathrm{v})$ in the ice cold mortar using $50 \mathrm{mM}$ Tris- $\mathrm{HCl}$ buffer $\mathrm{pH}$ 7.6 containing $1 \mathrm{mM}$ EDTA, $1 \mathrm{mM} \mathrm{MgCl}_{2}, 10 \mathrm{mM}$ $\beta$-mercaptoethanol, $1 \mathrm{mM}$ dithiothreitol, and $0.5 \%(\mathrm{~m} / \mathrm{v})$ polyvinylpyrrolidone (PVP). The homogenates were centrifuged at $20000 \mathrm{~g}$ for $20 \mathrm{~min}$.

Enzyme assays: The activity of PEPC was measured in a coupled enzymatic assay with malate dehydrogenase and was expressed in $\mu$ mol NADH oxidized per mg protein per minute as described by Gajewska et al. (2013). Nitrogen metabolism-related enzymes, i.e. NR, GS, Fd-GOGAT, GDH, ALT, and AST were determined according to the previously published protocols (Gajewska and Skłodowska 2009). NR activity was assayed by measuring the content of $\mathrm{NO}_{2}^{-}$and was expressed in nmol $\mathrm{NO}_{2}^{-}$formed per $\mathrm{mg}$ protein per

\section{Results}

In the inoculated leaves of $\mathrm{C}_{3}$ plants, $B$. cinerea conidia germination and the hyphal growth were facilitated when compared to CAM plants. The light micrographs taken $20 \mathrm{~h}$ after inoculation showed that within the inoculuminfiltrated areas in $\mathrm{C}_{3}$ leaves, the germinating spores formed long, proliferating hyphae filled with dense cytoplasm whereas in CAM plants, the germ tubes were short and swollen (Fig. 1).

The PEPC activity in control CAM M. crystallinum plants was several-fold higher than in $\mathrm{C}_{3}$ plants (Fig. 2). The difference was more pronounced in the $2^{\text {nd }}$ leaf pair showing on the $1^{\text {st }}$ day of experiment about 9-fold higher PEPC activity in CAM plants compared to $\mathrm{C}_{3}$ plants. At the same time in the $3^{\text {rd }}$ leaf pair of CAM plants, the activity of this enzyme was about 7-fold higher than in $\mathrm{C}_{3}$ plants. In both $\mathrm{C}_{3}$ and $\mathrm{CAM}$ plants, infection with $B$. cinerea resulted in an increase in PEPC activity in the $2^{\text {nd }}$ leaf pair ( $3^{\text {rd }}$ dai). Compared to the control, it was minute. The activation state of NR (reflecting how much of the enzyme is in the non-phosphorylated active form) was given as a percentage ratio between actual (measured in the presence of $\mathrm{Mg}^{2+}$ ) and maximum (measured in the presence of EDTA) NR activity. Fd-GOGAT activity was assayed with methyl viologen as electron donor and was expressed in nmol glutamate formed per mg protein per minute. GS activity was estimated using the transferase assay based on reaction of glutamine with hydroxylamine and was expressed in $\mu$ mol $\gamma$-glutamylhydroxamate formed per mg protein per minute. NADH-GDH and NAD-GDH activities were assayed spectrophotometrically by monitoring the oxidation of NADH or reduction of NAD and were expressed in nmol NADH oxidized or NAD reduced per mg protein per minute, respectively. ALT activity was assayed in the alanine $\rightarrow$ pyruvate direction by coupling the reaction with $\mathrm{NADH}$ oxidation by lactate dehydrogenase. AST activity was assayed in the aspartate $\rightarrow$ oxaloacetate direction by coupling the reaction with NADH oxidation by malate dehydrogenase. ALT and AST activities were expressed in $\mu$ mol NADH oxidized per mg protein per minute.

Protein content was determined according to Bradford (1976) using standard curves prepared for bovine serum albumin.

Data analysis: The data are means from 6 independent experiments $(n=6)$. In each experiment, two plants were used to prepare one leaf sample, and a single sample was analyzed for each treatment. Sample variability was given as a standard deviation ( \pm SD) of the mean. The significance of differences between control and infected plants as well as between $\mathrm{C}_{3}$ and CAM plants was determined by Student's $t$-test using the Statistica ${ }^{\circledR}$ software. Differences at $P<0.05$ were considered significant.

increased by 37 and $27 \%$ in $\mathrm{C}_{3}$ and CAM plants, respectively. In the non-inoculated $3^{\text {rd }}$ leaf pair as early as 1 dai both $\mathrm{C}_{3}$ and CAM plants showed induction of PEPC activity by 40 and $37 \%$, respectively. On the $3^{\text {rd }}$ dai the activity of this enzyme was increased only in CAM plants, by $58 \%$ over the control level.

In the $3^{\text {rd }}$ leaf pair, the control NR activity was significantly higher in CAM plants as compared to $\mathrm{C}_{3}$ plants (Fig. 3). In both $\mathrm{C}_{3}$ and CAM plants, infection led to a decline in NR activity in the $2^{\text {nd }}$ leaf pair. In $\mathrm{C}_{3}$ plants decreases in NR activity were observed on the $1^{\text {st }}$ and $3^{\text {rd }}$ dai by 36 and $42 \%$, respectively. In CAM plants a detrimental effect of infection on NR activity was visible starting from the $2^{\text {nd }}$ dai, when it was decreased by $26 \%$ compared to the control. On the $3^{\text {rd }}$ dai, the activity of this enzyme was reduced by $45 \%$. In the noninoculated $3^{\text {rd }}$ leaf pair, no significant changes in NR activity were observed in response to infection except for 
the $2^{\text {nd }}$ dai when in $\mathrm{C}_{3}$ plants it was increased by $46 \%$ compared to the control.

Infection did not significantly influence NR activation state in M. crystallinum leaves except for the $2^{\text {nd }}$ leaf pair of $\mathrm{C}_{3}$ plants which on the $3^{\text {rd }}$ dai showed its increase by $27 \%$ compared to the control (Fig. 3). The GS activities in control $2^{\text {nd }}$ and $3^{\text {rd }}$ leaf pairs of CAM plants were up to $20 \%$ lower than those in $\mathrm{C}_{3}$ plants (Fig. 4). Neither in $\mathrm{C}_{3}$ nor in CAM plants infection changed GS activity in leaves. Infection with $B$. cinerea differently influenced Fd-GOGAT activity in the $2^{\text {nd }}$ leaf pair of $\mathrm{C}_{3}$ and CAM plants (Fig. 4). Whereas $\mathrm{C}_{3}$ plants showed a $26 \%$ decrease in this enzyme activity on the $1^{\text {st }}$ dai and subsequently a $25 \%$ increase on the $3^{\text {rd }}$ dai, in CAM plants a $40 \%$ induction found on the $1^{\text {st }}$ dai was followed by a $31 \%$ inhibition on the $2^{\text {nd }}$ dai. In the non-inoculated $3^{\text {rd }}$ leaf pair, an infection-induced $50 \%$ increase in Fd-GOGAT activity was observed in CAM plants on the $1^{\text {st }}$ dai.

The $\mathrm{C}_{3}$ plants did not show significant changes in NADH-GDH activity in response to infection (Fig. 5). On the contrary, in CAM plants, infection resulted in a decrease in NADH-GDH activity on the $1^{\text {st }}$ dai, both in the $2^{\text {nd }}$ and $3^{\text {rd }}$ leaf pairs, by 31 and $44 \%$ compared to the
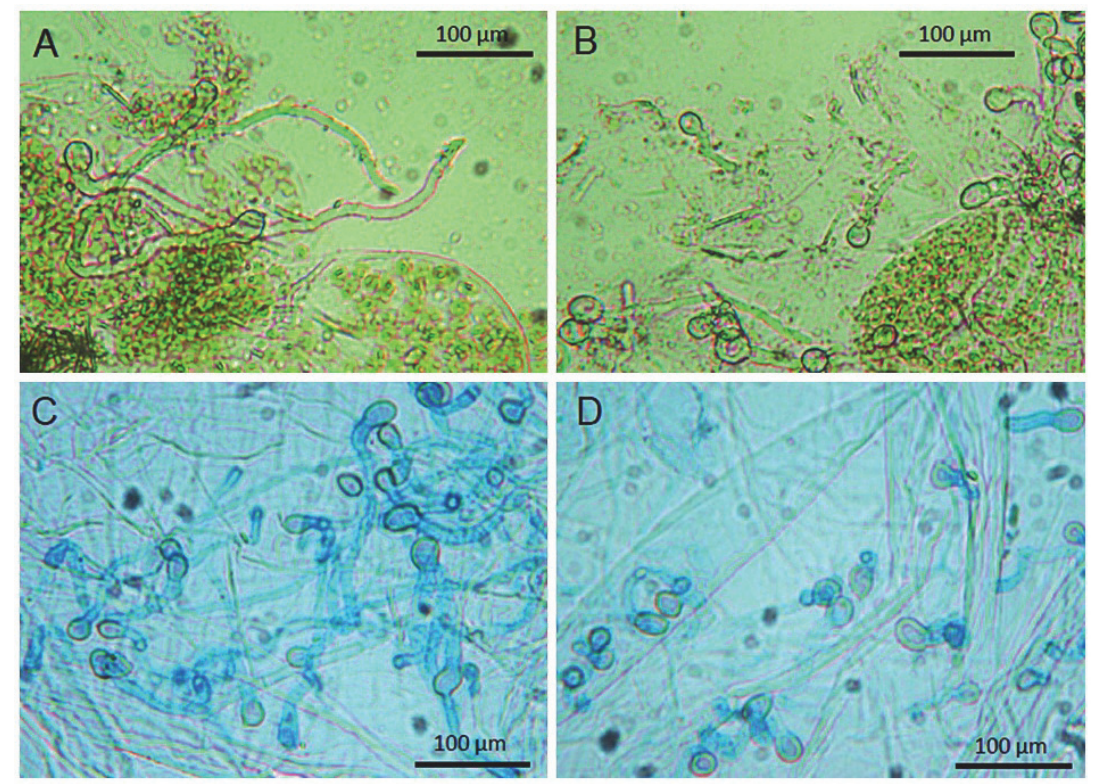

Fig. 1. Botrytis cinerea conidia germination and hyphae growth in infected Mesembryanthemum crystallinum leaves (the $2^{\text {nd }}$ pair). Light micrographs were taken $20 \mathrm{~h}$ after inoculation. $A$ and $C-C_{3}$ plants; $B$ and $D$ - CAM plants; Trypan blue staining $(C, D)$. Images are representative of three independent experiments using a minimum of three $\mathrm{C}_{3}$ and CAM plants.

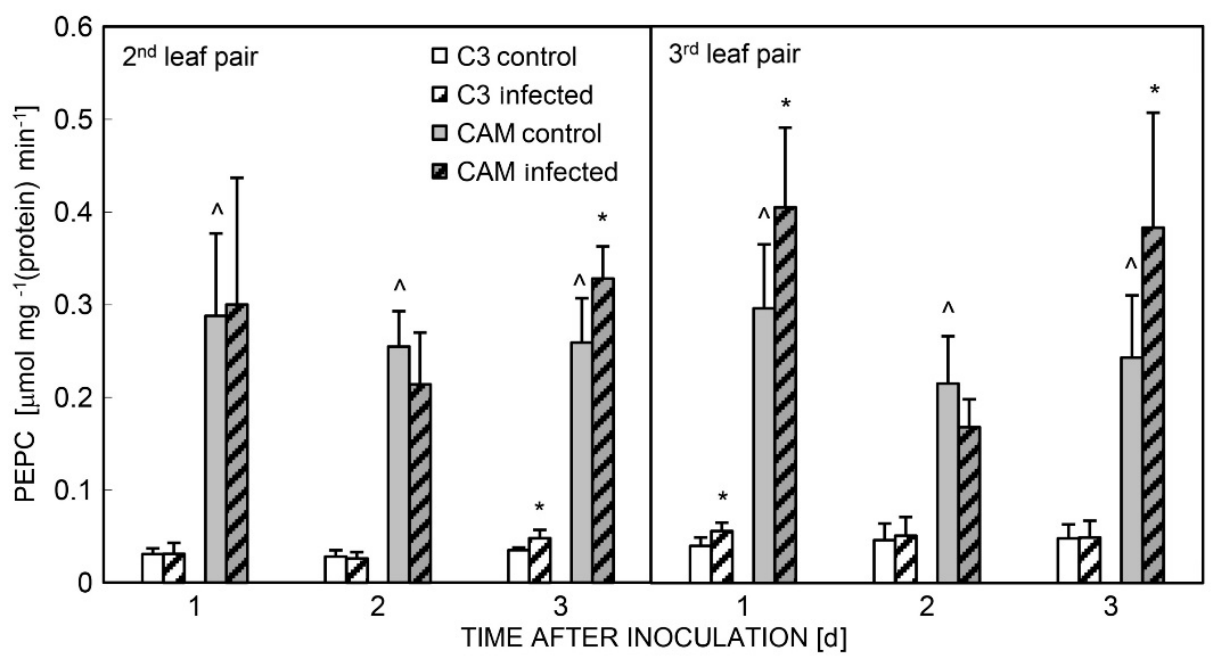

Fig. 2. Effect of Botrytis cinerea infection on phosphoenolpyruvate carboxylase (PEPC) activity in the inoculated $2^{\text {nd }}$ and noninoculated $3^{\text {rd }}$ leaf pairs of $\mathrm{C}_{3}$ and CAM Mesembryanthemum crystallinum plants. Means $\pm \mathrm{SDs}, n=6,{ }^{*}$ indicate a significant difference between control and infected plants at $P<0.05,{ }^{\wedge}$ indicate a significant difference between $\mathrm{C}_{3}$ and $\mathrm{CAM}$ plants at $P<0.05$. 
control, respectively. On the $3^{\text {rd }}$ dai an increase in NADH-GDH was found only in the $2^{\text {nd }}$ leaf pair, by $37 \%$ compared to the control. The NAD-GDH activity (deaminating) was about 2 to 4-fold higher than NADH-GDH activity (aminating), depending on the leaf age and type of metabolism (Fig. 5). Irrespective of the leaf age, the control NAD-GDH activity in CAM plants was over 2-fold higher compared to $\mathrm{C}_{3}$ plants. Infection did not significantly alter NAD-GDH activity in M. crystallinum leaves.

The control $\mathrm{C}_{3}$ plants exhibited significantly higher ALT activity compared to CAM plants, both in the $2^{\text {nd }}$ and $3^{\text {rd }}$ leaf pairs (Fig. 6). Infection of $\mathrm{C}_{3}$ plants resulted in an increase in ALT activity in the $2^{\text {nd }}$ leaf pair on the $1^{\text {st }}$ and the $2^{\text {nd }}$ dai, by 33 and $39 \%$ over the control, respectively. The $2^{\text {nd }}$ leaf pair of CAM plants showed a $44 \%$ increase in ALT activity on the $3^{\text {rd }}$ dai. The ALT activity in the $3^{\text {rd }}$ leaf pair in both $\mathrm{C}_{3}$ and CAM plants was not influenced by $B$. cinerea infection.

Compared to ALT, AST exhibited higher activity in control plants (Fig. 6). The $2^{\text {nd }}$ leaf pair of $\mathrm{C}_{3}$ and CAM plants responded differently to infection, the former showing a $25 \%$ decrease in AST activity on the $1^{\text {st }}$ dai and the latter a $44 \%$ increase on the $3^{\text {rd }}$ dai. The activity of AST in the non-inoculated $3^{\text {rd }}$ leaf pair of $\mathrm{C}_{3}$ plants increased on the $2^{\text {nd }}$ dai while in those of CAM plants on the $3^{\text {rd }}$ dai, by $34 \%$ and $43 \%$ compared to the control, respectively.

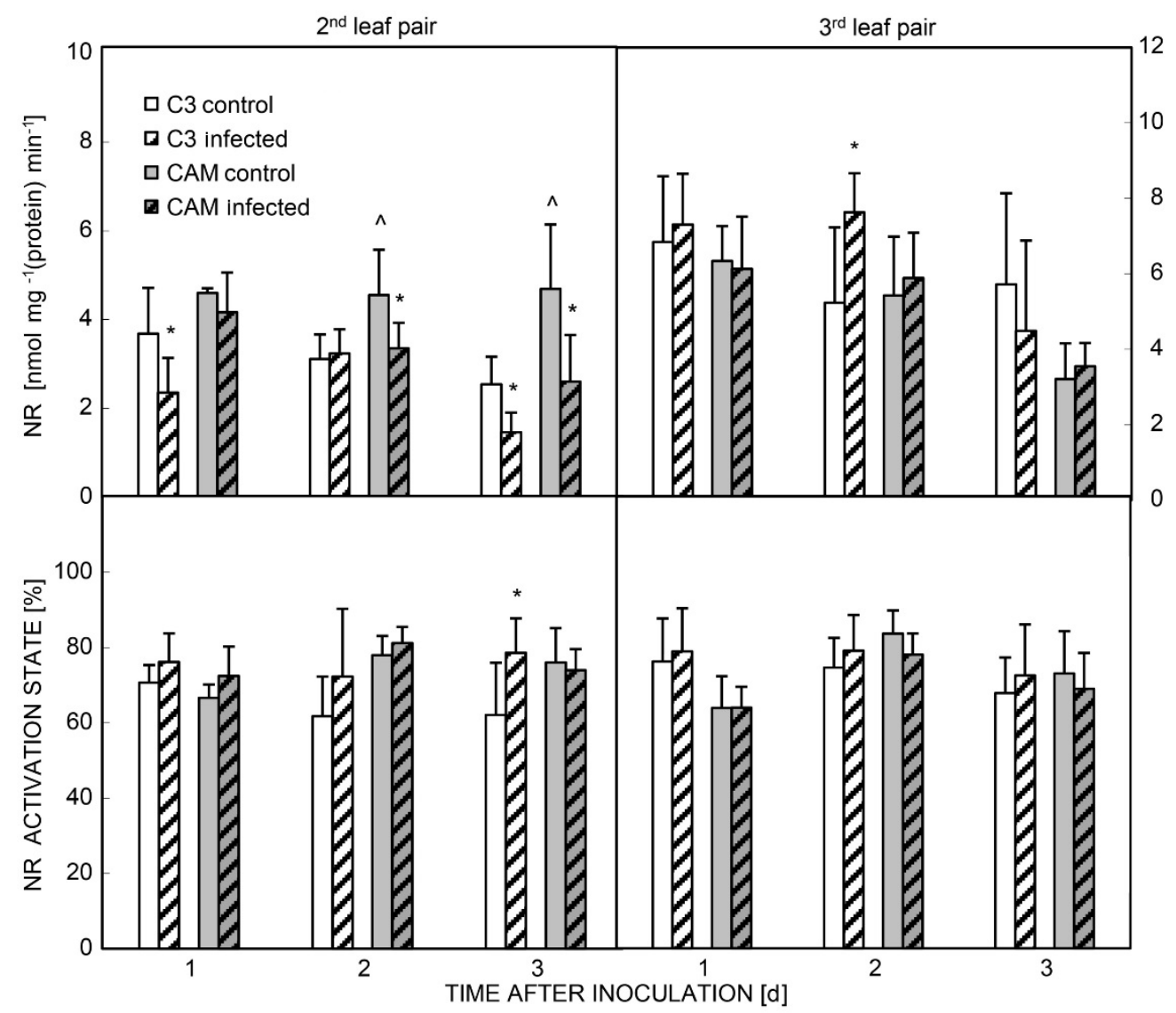

Fig. 3. Effect of Botrytis cinerea infection on nitrate reductase (NR) activity and NR activation state in the inoculated $2^{\text {nd }}$ and noninoculated $3^{\text {rd }}$ leaf pairs of $\mathrm{C}_{3}$ and CAM Mesembryanthemum crystallinum plants. Means $\pm \mathrm{SDs}, n=6$, $*$ indicate a significant difference between control and infected plants at $P<0.05, \wedge$ indicate a significant difference between $\mathrm{C}_{3}$ and $\mathrm{CAM}$ plants at $P<0.05$.

\section{Discussion}

Our earlier studies showed that both C3 and CAM M. crystallinum plants are resistant to B. cinerea. Within $48 \mathrm{~h}$ after inoculation, symptom development is facilitated in $\mathrm{C} 3$ plants, however, the further $B$. cinerea growth is restricted within necrotic lesions resulting from a hypersensitive-like resistance response in plants representing both $\mathrm{C} 3$ and $\mathrm{CAM}$ metabolism types
(Kuźniak et al. 2010, Libik-Konieczny et al. 2011). We found that $\mathrm{NaCl}$ in the concentration used for CAM induction influences the $B$. cinerea hyphae morphology (Kuźniak et al., 2011). However, the restriction of symptom development is probably not due to $\mathrm{NaCl}$ action as it occurs in both $\mathrm{C} 3$ and CAM plants. Moreover, $\mathrm{NaCl}$ in the concentration range from 0.1 to $0.8 \mathrm{M}$, does not 
inhibit B. cinerea growth in vitro (Kuźniak et al. 2010).

As photosynthetic carbon metabolism is intimately linked with nitrogen metabolism (Nunes-Nesi et al. 2010), and modulation of enzymes of carbon and nitrogen metabolism could be an important regulatory point of carbon/nitrogen interaction, the present study addressed changes in activities of PEPC and nitrogen metabolism enzymes in $\mathrm{C}_{3}$ and CAM M. crystallinum plants after $B$. cinerea infection. In our study, PEPC activity in the leaves of CAM plants was several-fold higher than in $\mathrm{C}_{3}$ plants. Compared to $\mathrm{C}_{3}$ plants, those performing CAM showed significantly higher activities of NR and NAD-GDH but lower activities of GS and ALT which could reflect CAM-specific changes in the $\mathrm{C} / \mathrm{N}$ status of plant cells. Increased activity of NR observed in some halophytic species grown under saline conditions has been attributed to high nitrogen requirements arising from the accumulation of nitrogenous osmoprotectants, such as proline or glycine betaine, in their tissues (Stewart and Rhodes 1978). Despite difference in the control level of NR activity, $\mathrm{C}_{3}$ and CAM $M$. crystallinum plants showed a similar activation state of this enzyme.

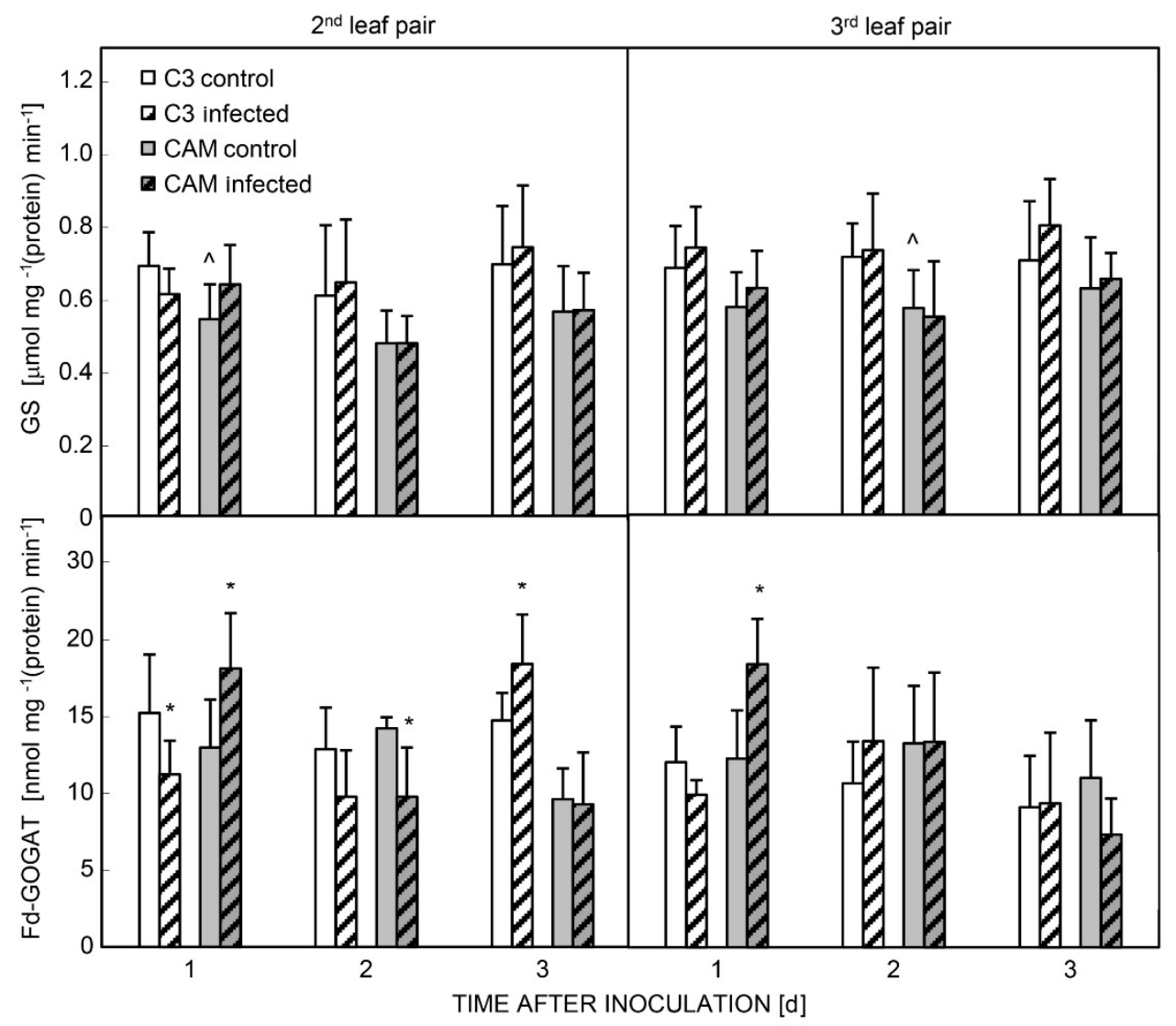

Fig. 4. Effect of Botrytis cinerea infection on glutamine synthetase (GS) and ferredoxin-dependent glutamate synthase (Fd-GOGAT) activities in the inoculated $2^{\text {nd }}$ and non-inoculated $3^{\text {rd }}$ leaf pairs of $\mathrm{C}_{3}$ and CAM Mesembryanthemum crystallinum plants. Means $\pm \mathrm{SDs}, n=6, *$ indicate a significant difference between control and infected plants at $P<0.05, \wedge$ indicate a significant difference between $\mathrm{C}_{3}$ and CAM plants at $P<0.05$.

In $\mathrm{C}_{3}$ and especially in CAM plants, the activity of NAD-GDH markedly exceeded that of NADH-GDH indicating that glutamate dehydrogenase served mainly catabolic function, as it is reported for majority of higher plants (Lea and Miflin 2003). Higher NAD-GDH activity in control CAM plants than in $\mathrm{C}_{3}$ plants reflected intensive glutamate deamination resulting in the release of 2-oxoglutarate. This reaction can be used to funnel the $\mathrm{C}$ skeleton of glutamate into the TCA cycle.

Despite different PEPC activity in control $\mathrm{C}_{3}$ and CAM $M$. crystallinum plants, they responded similarly to $B$. cinerea infection showing an increase in this enzyme activity on the $3^{\text {rd }}$ dai. These results indicate that in plants challenged by the pathogen, regardless of the type of photosynthetic carbon metabolism, $\beta$-carboxylationrelated processes could be involved in reprogramming plant metabolism from growth and development to defense (Doubnerová and Ryšlavá 2011). Besides providing $\mathrm{CO}_{2}$ for Calvin cycle, the infection-inducible PEPC promotes the anaplerotic replenishment of $\mathrm{C}_{4}$-dicarboxylic acids utilized for energy and biosynthetic metabolism which could be advantageous for plants under biotic stress. Comparing to our findings a much higher increase in PEPC activity was reported for plants subjected to viral infection (Ryšlavá et al. 2003).

Regardless of the mode of photosynthesis, the 
infected leaves showed a significant decrease in NR activity, however, the pattern of changes depended on the type of metabolism performed. Inhibitory effect of infection on NR activity was observed earlier in $\mathrm{C}_{3}$ plants, which might contribute to the faster leaf colonization by $B$. cinerea. The observed decrease in NR activity seems to be unrelated to the post-translational modifications of the enzyme molecule by phosphorylation, which was evidenced by unchanged or even increased NR activation state found in $B$. cinereainfected leaves. Besides decline in enzyme synthesis or its increased degradation (Botrel and Kaiser 1997), a decrease in maximum NR activity found in our study might have resulted from the shortage of a reductant in infected $M$. crystallinum leaves. Nitrate assimilation is sensitive to the availability of NADH, which comes, among others, from the photosynthetic electron transport chain (Foyer and Noctor 2002). We found that in $\mathrm{C}_{3}$ plants, the photochemical activity remained unchanged after B. cinerea infection whereas in infected leaves of CAM plants significant decreases in maximal photosystem II quantum yield (characterized by variable to maximum chlorophyll fluorescence ratio, $\mathrm{F}_{\mathrm{v}} / \mathrm{F}_{\mathrm{m}}$ ), photochemical quenching (qP), and non-photochemical quenching (NPQ) were observed 3 dai (Gabara et al. 2012). We suggest that the depressed photochemical activity may be important for limiting nitrogen assimilation via NR in infected CAM leaves found in this study. The infection-induced NR activity decrease in both $\mathrm{C}_{3}$ and CAM plants could be, at least 3 dai when PEPC activity increased, the effect of feedback regulation of NR by malate. As shown by Müller et al. (2001), malate represses NR at the transcription and enzyme activity levels but does not affect enzyme activation via reversible phosphorylation. However, NR activity increase in the non-inoculated $3^{\text {rd }}$ leaf pair of infected $\mathrm{C}_{3}$ plants 2 dai could reflect the stimulatory effect of photorespiration on nitrate reduction (Nunes-Nesi et al. 2010).

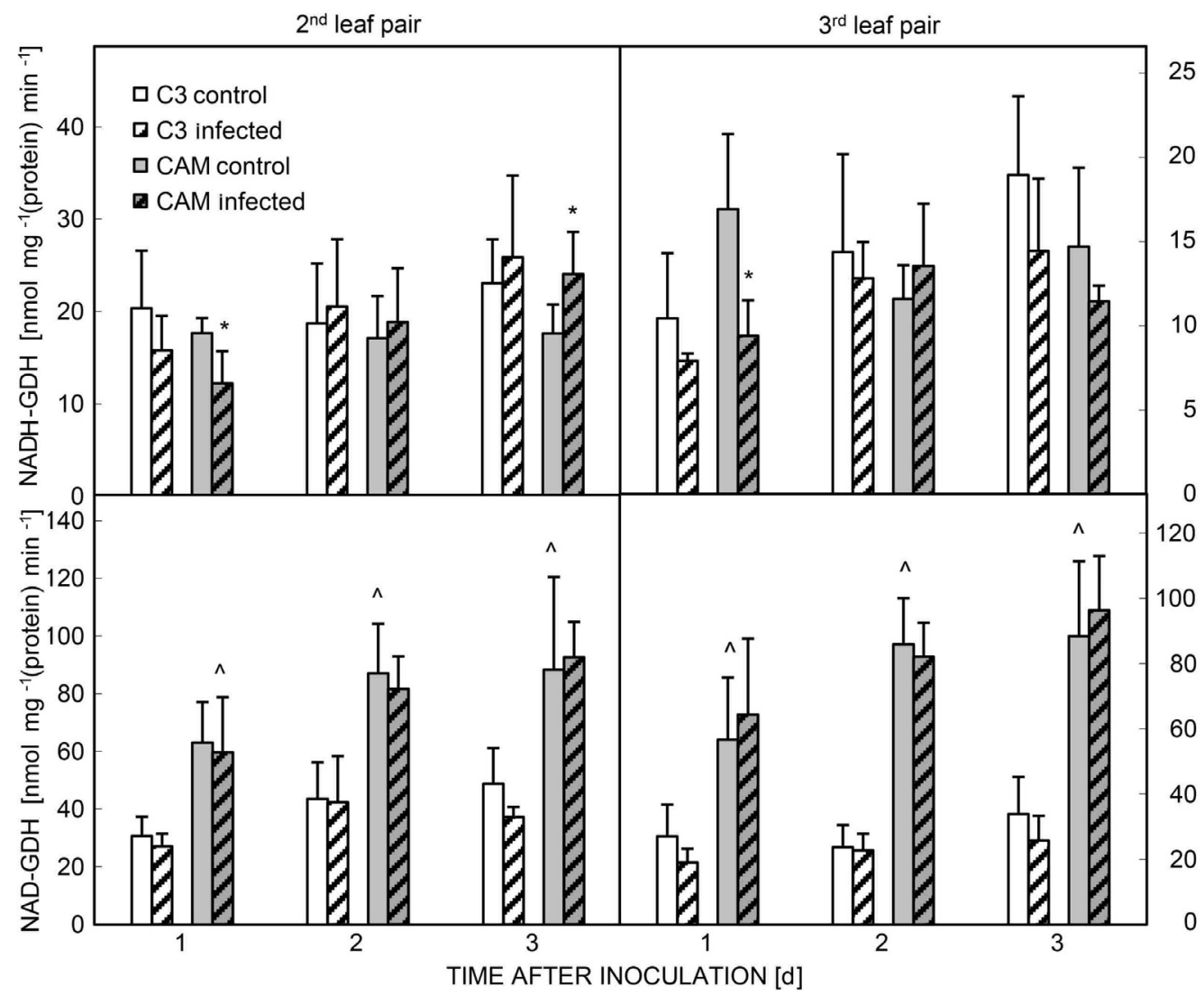

Fig. 5. Effect of Botrytis cinerea infection on NADH-dependent glutamate dehydrogenase (NADH-GDH) and NAD-dependent glutamate dehydrogenase (NAD-GDH) activities in the inoculated $2^{\text {nd }}$ and non-inoculated $3^{\text {rd }}$ leaf pairs of $C_{3}$ and CAM Mesembryanthemum crystallinum plants. Means $\pm \mathrm{SDs}, n=6$, * indicate a significant difference between control and infected plants at $P<0.05, \wedge$ indicate a significant difference between $\mathrm{C}_{3}$ and CAM plants at $P<0.05$.

Amino acids and amino acid-derived metabolites play multiple roles in plant resistance to pathogens (Liu et al. 2010, Zeier 2013). The depletion of amino acids, especially glutamine, enhances defense reactions in Arabidopsis (Liu et al. 2010). As in our study, the
GS activity in the inoculated $2^{\text {nd }}$ leaves remained unchanged, one can speculate that this might have helped to limit $B$. cinerea infection. In accordance with the postulated key role of glutamate in plant-pathogen interactions (Seifi et al. 2013), we found infection- 
induced changes in activities of all glutamate-yielding enzymes, namely ALT, AST, NADH-GDH, and Fd-GOGAT. In both $\mathrm{C}_{3}$ and CAM plants, increased Fd-GOGAT activity was concomitant with $B$. cinerea restriction in infected leaves. The increased Fd-GOGAT activity in leaves of infected CAM plants found 1 dai, when the spore germinating rate slowed down, could indicate the importance of glutamate recycling shortly after inoculation for the defense strategy against B. cinerea. In line with this assumption, in inoculated $\mathrm{C}_{3}$ leaves, $B$. cinerea invasion was restricted later (2 - 3 dai) (Kuźniak et al. 2010) and Fd-GOGAT induction was observed only 3 dai. The potent requirements for glutamate recycling in infected leaves could also be met by aminotransferases operating in the catabolic direction. The involvement of ALT and AST depended on the type of photosynthetic carbon metabolism, however, in both $\mathrm{C}_{3}$ and CAM plants their induction compensated for the decrease in Fd-GOGAT activity in the course of infection. In the inoculated leaves of $\mathrm{C}_{3}$ plants, ALT was significantly increased 1 and 2 dai whereas in CAM plants, ALT and AST were induced only 3 dai. The enhanced ALT activity supported alanine catabolism in cells of infected $\mathrm{C}_{3}$ leaves undergoing B. cinerea-induced programmed cell death (Kuźniak et al. 2013). Given the absence of programmed cell death events in CAM plants (Kuźniak et al. 2013), the increased catabolism of alanine and aspartate found in these plants 3 dai may provide pyruvate and oxaloacetate for the TCA cycle to funnel the energy-demanding metabolic pathways. Besides their potential anaplerotic functions, aminotransferases can also be crucial for activation of resistance against pathogens, as shown for specific Arabidopsis aminotransferase generating a putative amino acid-derived signal mediating multicomponent local and systemic defense responses (Song et al. 2004). Moreover, AST provides substrate oxaloacetate for phosphoenolpyruvate carboxykinase, which besides being involved as decarboxylase in $\mathrm{CO}_{2}$ concentrating mechanism in CAM plants, plays a role in nitrogen metabolism (Leegood and Walker 2003, Delgado-Alvarado et al. 2007)

As adequate metabolic adjustment at the whole organism level is crucial for plant survival under stress, it can be assumed that metabolic information on nitrogen status of the infected leaf is transferred between organs and drives metabolic adjustments, e.g., carbon/nitrogen balance, in non-inoculated parts of the plant (Coruzzi and Zhou 2001, Rojas et al. 2014). In leaves, NADH-NR and PEPC along with sucrose phosphate synthetase were

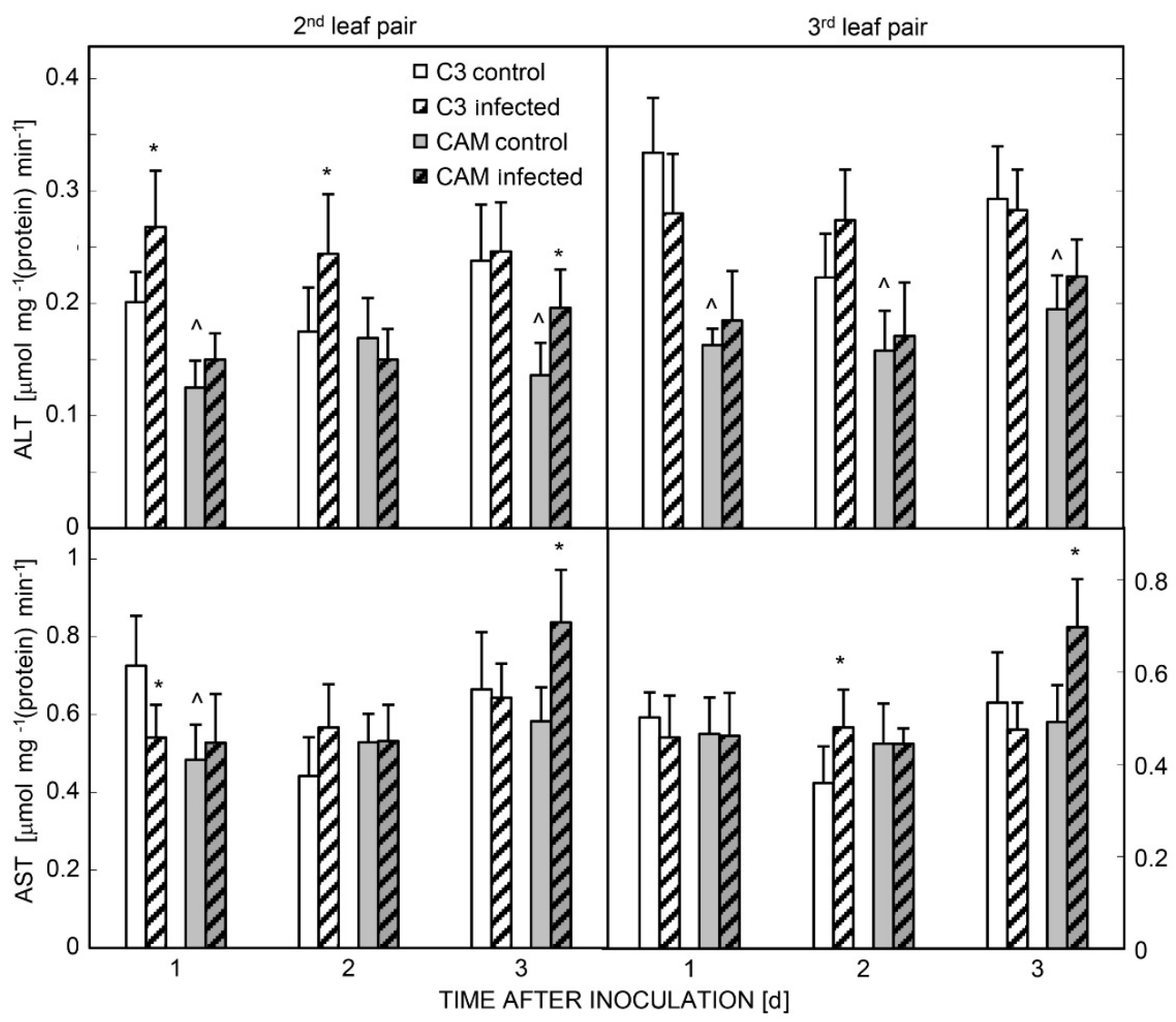

Fig. 6. Effect of Botrytis cinerea infection on alanine aminotransferase (ALT) and aspartate aminotransferase (AST) activities in the inoculated $2^{\text {nd }}$ and non-inoculated $3^{\text {rd }}$ leaf pairs of $\mathrm{C}_{3}$ and CAM Mesembryanthemum crystallinum plants. Means \pm SDs, $n=6$, $*$ indicate a significant difference between control and infected plants at $P<0.05$, ^ indicate a significant difference between $\mathrm{C}_{3}$ and CAM plants at $P<0.05$. 
postulated to be important in carbon and nitrogen interaction (Champigny 1995). We found that in infected CAM plants, changes in activities of Fd-GOGAT and NADH-GDH on the $1^{\text {st }}$ dai as well as in PEPC and AST on the $3^{\text {rd }}$ dai induced in the inoculated $2^{\text {nd }}$ leaf pair were mirrored in the non-inoculated $3^{\text {rd }}$ leaf pair. However, in $\mathrm{C}_{3}$ plants, the responses of inoculated and non-inoculated leaves were not parallel as far as PEPC, AST, and NR activity changes were analyzed. Enzyme activity changes found in the non-inoculated $3^{\text {rd }}$ leaf pair of infected $\mathrm{C}_{3}$ plants were not observed in the inoculated $2^{\text {nd }}$ leaf pair (AST, NR), or they exhibited a different time course (PEPC). Nevertheless, these results suggest that local infection changed the carbon/nitrogen status in healthy upper leaves by mechanisms involving PEPC and enzymes of nitrogen metabolism to a degree which was specific to the type of photosynthetic $\mathrm{CO}_{2}$ assimilation. Given the location of PEPC, AST (Aubry et al. 2011), and Fd-GOGAT (Chang et al. 2012) in bundle sheath cells, and the role of vascular tissues in systemic stress responses in plants, including biotic stress (Vlot et al. 2008), activity changes of these enzymes found in healthy leaves of infected plants may be important in maintenance the carbon/nitrogen balance throughout the plant. In stressed plants, these changes could contribute to adjustment to a new state of metabolic homeostasis referred as acclimation. It is an attractive possibility that besides being involved in basal carbon and nitrogen

\section{References}

Aubry, S., Brown, N.J., Hibberd, J.M.: The role of proteins in $\mathrm{C}(3)$ plants prior to their recruitment into the $\mathrm{C}(4)$ pathway. - J. exp. Bot. 62: 3049-3059, 2011.

Bilgin, D.D., Zavala, J., Zhu, J., Clough, S.J., Or, D.R., DeLucia, E.H.: Biotic stress globally downregulates photosynthesis genes. - Plant Cell Environ. 33: 1597-1613, 2010.

Bolton, M.D., Thomma, B.P.H.J.: The complexity of nitrogen metabolism and nitrogen-regulated gene expression in plant pathogenic fungi. - Physiol. mol. Plant Pathol. 72: 104-110, 2008.

Botrel, A., Kaiser, W.M.: Nitrate reductase activation state in barley roots in relation to the energy and carbohydrate status. - Planta 201: 496-501, 1997.

Bradford, M.M.: A rapid and sensitive method for the quantitation of microgram quantities of protein utilizing the principle of protein-dye binding. - Anal. Biochem. 72: 248254, 1976.

Cebeci, O., Kokturk, B., Ergen, N., Ozturk, L., Cakmak, I., Budak, H.: Differential expression of wheat transcriptomes in response to varying cadmium concentrations. - Biol. Plant. 52: 703-708, 2008.

Champigny, M.L.: Integration of photosynthetic carbon and nitrogen metabolism in higher plants. - Photosynth. Res. 46: 117-127, 1995.

Chang, Y.-M., Liu, W.-Y., Shih, A.C.-C., Shen, M.-N., Lu, C.H., Lu, M.-Y.J., Yang, H.-W., Wang, T.-Y., Chen, S.C.-C., Chen, S.M., Li, W.-H., Ku, M.S.B.: Characterizing regulatory and functional differentiation between maize mesophyll and bundle sheath cells by transcriptomic metabolism, PEPC and enzymes of nitrogen metabolism may play a role in the establishment of whole plant response to infection, as suggested for PEPC in Arabidopsis subjected to abiotic stresses (Sánchez et al. 2006). It cannot be excluded, however, that activity changes in nitrogen metabolism-related enzymes found in the non-inoculated leaves of infected plants resulted from infection-triggered changes in allocation of nitrogen resources, which were affected by the type of photosynthetic carbon metabolism (Schultz et al. 2013).

In conclusion, our results indicate that the CAMspecific post-inoculation changes manifested by earlier increase in PEPC and Fd-GOGAT activities as well as later inhibition of NR activity could contribute to faster restriction of $B$. cinerea growth found in the infected leaves of those plants. Moreover, in CAM-performing plants, the activities of PEPC, Fd-GOGAT, NADH-GDH, and AST in the non-inoculated $3^{\text {rd }}$ leaf pair were similarly influenced by the pathogen as in the inoculated $2^{\text {nd }}$ leaf pair. This points to CAM-specific, local infectioninduced alteration of carbon/nitrogen status in systemic leaves resulting from changes in PEPC and nitrogen metabolism-related enzymes. As this relationship was not observed in $\mathrm{C}_{3}$ plants where $B$. cinerea growth was somehow facilitated, it can be suggested that the CAMspecific pattern of changes in nitrogen metabolismrelated enzymes favours the expression of defense mechanisms. analysis. - Plant Physiol. 160: 165-177, 2012.

Coruzzi, G.M., Zhou, L.: Carbon and nitrogen sensing and signaling in plants: emerging "matrix effects". - Curr. Opin. Plant Biol. 4: 247-253, 2001.

Delgado-Alvarado, A., Walker, R.P., Leegood, R.C.: Phosphoenolpyruvate carboxykinase in developing pea seeds is associated with tissues involved in solute transport and is nitrogen-responsive. - Plant Cell Environ. 30: 225235, 2007.

Doubnerová, V., Ryšlavá, H.: What can enzymes of $\mathrm{C}_{4}$ photosynthesis do for $\mathrm{C}_{3}$ plants under stress? - Plant Sci. 180: 575-583, 2011.

Foyer, C.H., Noctor, G.: Photosynthetic nitrogen assimilation: inter-pathway control and signaling. - In: Foyer, C.H., Noctor, G., (ed.): Photosynthetic Nitrogen Assimilation and Associated Carbon and Respiratory Metabolism, Advances in Photosynthesis and Respiration. Pp. 1-22. Kluwer Academic Publishers, Dordrecht 2002.

Gabara, B., Kuźniak, E., Skłodowska, M., Surówka, E., Miszalski, Z.: Ultrastructural and metabolic modifications at the plant-pathogen interface in Mesembryanthemum crystallinum leaves infected by Botrytis cinerea. - Environ. exp. Bot. 77: 33-43, 2012.

Gajewska, E., Niewiadomska, E., Tokarz, K., Słaba, M., Skłodowska, M.: Nickel-induced changes in carbon metabolism in wheat shoots. - J. Plant Physiol. 170: 369377, 2013.

Gajewska, E., Skłodowska, M.: Nickel-induced changes in nitrogen metabolism in wheat shoots. - J. Plant Physiol. 166: 1034-1044, 2009. 